\title{
Mobile Device Based Personalized Equalizer for Improving Hearing Capability of Human Voices in Particular for Elderly Persons
}

\author{
Kohei Arai 1 \\ Graduate School of Science and Engineering \\ Saga University \\ Saga City, Japan
}

\author{
Takuto Konishi 1 \\ Graduate School of Science and Engineering \\ Saga University \\ Saga City, Japan
}

\begin{abstract}
Mobile device based personalized equalizer for improving the hearing capability of human voices in particular for elderly persons are proposed. Through experiments, it is found that the proposed equalizer does work well for improving hearing capability by 2 to $55 \%$ of voice Recognition success ratio. According to the investigation of the frequency component analysis and formant detections, most of the voice sounds have the formant frequencies for the first to third frequencies within the range of $3445 \mathrm{~Hz}$. Therefore, a nonlinear equalizing multiplier is better to enhance the frequency components for the first to third formants in particular. The experimental results with the voice above input experiments show that a good Percent Correct Recognition: PCR is required for 0 to more than $8000 \mathrm{~Hz}$ of frequency components. Also, $8162 \mathrm{~Hz}$ cut off frequency would be better for both noise suppressions and keeping a good PCR
\end{abstract}

Keywords-Frequency response equalization; mobile devices; formount frequancy; hearing capability; hearing aids

\section{INTRODUCTION}

In general, hearing capability of human voices is getting bad for elderly persons due to the fact that a high-frequency response of elderly persons' ears is getting poor. Hearing capability is defined with the well-known averaged hearing capability level that is defined as Averaged value of hearing capability for human voices regarding frequency components ranged from $500 \mathrm{~Hz}$ to $4000 \mathrm{~Hz}$. In accordance with the definition, 25-40 dB of loudness of human voices are difficult to hear slightly when human voice is not loud while 40-70 dB of loudness of human voices are difficult to hear when human voice is normal level.

Earlier devices, known as ear trumpets or ear horns [1], [2], were passive funnel-like amplification cones designed to gather sound energy and direct it into the ear canal. After that not so small number of methods have been proposed so far [3] $-[10]$

Although general purpose of frequency equalizer which allows compensation of hearing capability is used for that purpose (improvement of hearing capability), degradation of high frequency response depends on person. Therefore, customization is highly required for the frequency equalization devices. On the other hand, such customized frequency equalization devices are not so cheap and also are not so good looking. Therefore, frequency equalizing devices are not so popular.

The human voice has base frequency sounds and overtone sounds. Frequency components of human voices consist of formant $^{1}$ (peaks of frequency components which are used for characterization of personal human voices). Lower-frequency components are dominant for vowels, in general, while relatively higher-frequency components are dominant for consonants. For elderly persons, high-frequency components are getting difficult to hear which results in the consonants are getting difficult to hear. Such difficulties are depending on persons by persons. Therefore, it is not so easy to customize frequency equalizers which allow improvement of hearing capability, in particular, for elderly persons.

There are several ways of evaluating how well a hearing aid compensates for hearing loss. One approach is audiometry which measures a subject's hearing levels in laboratory conditions. The threshold of audibility for various sounds and intensities is measured in a variety of conditions. Although audiometric tests may attempt to mimic real-world conditions, the patient's experiences may differ. An alternative approach is self-report assessment of which the patient reports their experiences with the hearing aid in concern. The evaluation method proposed here is based on using Electroencephalogram : EEG sensor. Namely, in accordance with hearing quality, Peak Alpha Frequency: PAF amplitude is getting large while it is getting small when hearing quality is getting poor.

The following section describes the proposed method and implementation of the compensation filter including mobile devices followed by some experiments for a specific person. Then a conclusion is described together with some discussions.

\section{PROPOSED METHOD AND IMPLEMENTATION}

In order to create a new personalized frequency equalizer, mobile devices are used. Mobile devices with headsets or ear phones are getting cheap and are good looking as well. Therefore, users can carry the proposed personalized equalizer.

In order to characterize hearing capability degradation, a specific user has to try to hear some sentences which cover the

\footnotetext{
${ }^{1}$ http://newt.phys.unsw.edu.au/jw/formant.html
} 
spectral range from zero (Direct Current: DC) to around 20 $\mathrm{KHz}$ includes all the vowels and the consonants together with their overtones. Then a spectral response of a compensation filter for the specific user is designed. The compensation filter is implemented in a mobile device such as Android tablet terminal, i-phone, smart phone, etc.

Frequency responses of ears against human voices are, in general, characterized with formants which are shown in Fig.1. Namely, human voice spectra have peaks which are named as formants.

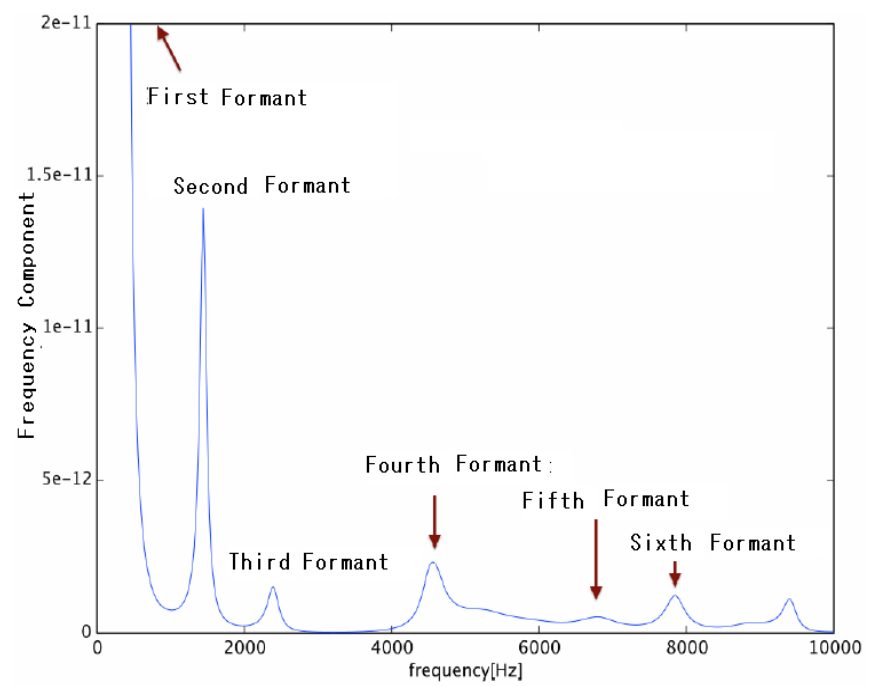

Fig. 1. Example of formants of human voices

According to the frequencies of the peaks, they are named the first formant, the second formant and so on. The proposed method detect formants through Forier Transformation first. In the mean time, input voice signals are decomposed with 32 of filter bank. Degraded formants can be found by comparing the input voice signals with the synthesized voice signals derived from Auditory Toolbox, for instance. Then the degraded formants can be compensated in accordance with the difference between actual voice and synthesized voice signals.

After that, reconstruction is applied to the degraded voice signals with 32 filter bank as shown in Fig.2. Delay time which is caused by the nonlinear equalizing multiplier can be compensated with deley element of which delay time is totally corresponding to the delay time caused by the nonlinear equalizing multiplier as shown in Fig.3

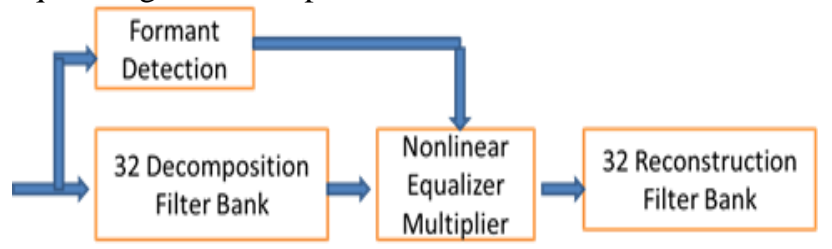

Fig. 2. Proposed method for degraded formant corrections with the consideration of formant ballunce

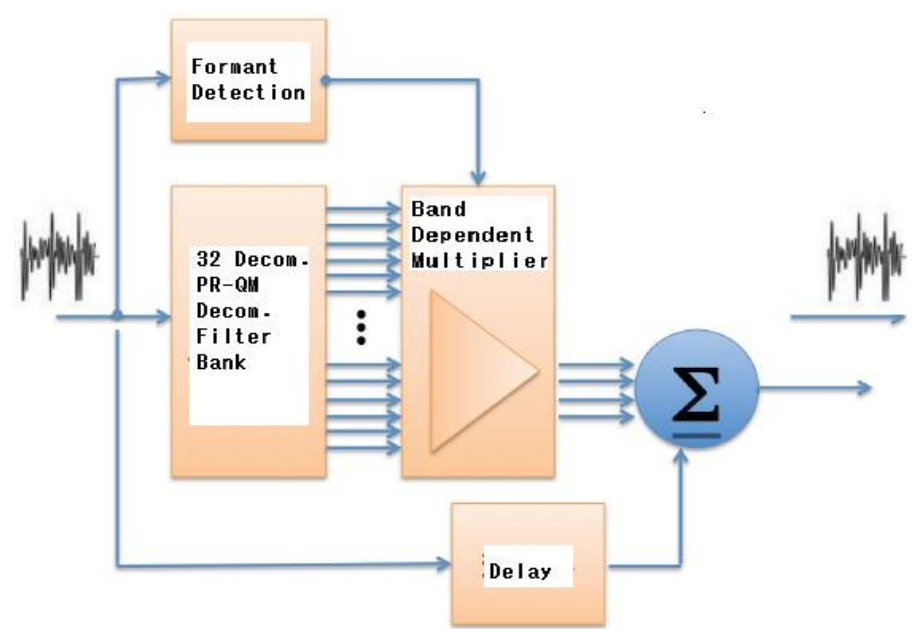

Fig. 3. Alternative of nonlinear equalizing multiplier with delay element

The correction filter is composed with hgh shelving filter of which the Frequency Transfer Function in analog filter function is expressed as follows,

$$
H(s)=A \frac{A s^{2}+\frac{\sqrt{A}}{Q} s+1}{S^{2}+\frac{\sqrt{A}}{Q} s+A}
$$

This can be re-written as follows,

$$
H(z)=\frac{b_{0}+b_{1} z^{-1}+b_{2} z^{-2}}{a_{0}+a_{1} z^{-1}+a_{2} z^{-2}}
$$

$$
\left\{\begin{array}{l}
b_{0}=A\left((A+2)+(A-2) \cos \omega_{0}+2 \sqrt{A} \alpha\right) \\
b_{1}=2 A\left((A-2)+(A+2) \cos \omega_{0}\right) \\
b_{2}=A\left((A+2)+(A-2) \cos \omega_{0}-2 \sqrt{A} \alpha\right) \\
a_{0}=(A+2)-(A-2) \cos \omega_{0}+2 \sqrt{A} \alpha \\
a_{1}=-2\left((A-2)-(A+2) \cos \omega_{0}\right) \\
a_{2}=(A+2)+(A-2) \cos \omega_{0}-2 \sqrt{A} \alpha
\end{array}\right.
$$

where $\alpha=\sin \omega_{0} / Q 、 \omega_{0}=2 \pi f_{0} / F_{s}$

The high shelving filter allows enhancement of arbitrary higher frequency components without suppression of low frequency components as shown in Fig.4. Also, the modulation transfer function of the high shelving filter is easy to design. Therefore, it is applicable for nonlinear equalizing multiplier.

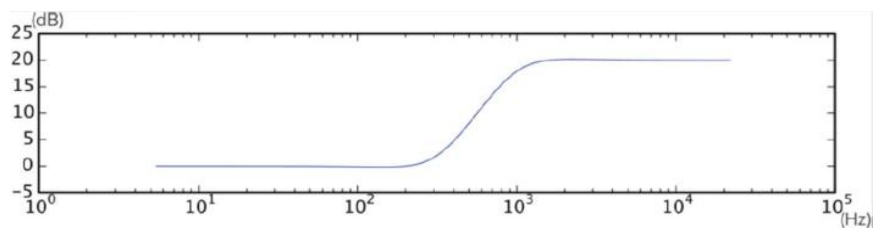

Fig. 4. Modulation Transfer Function of the high shelving filter 
When the high shelving filter is applied to the input voice signals, high frequency components are enhanced as shown in Fig.5.
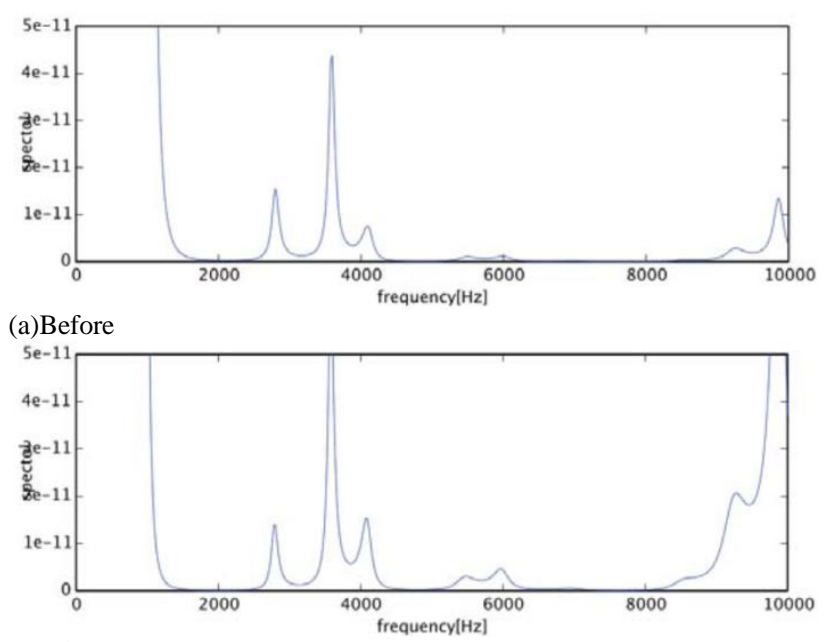

(b)After

Fig. 5. High frequency component enhancing nonlinear equalizing multiplier

Lastly, low pass filter is applied to the nonlinear equalizing multiplier applied voice signals for noise removal as shown in Fig.6.

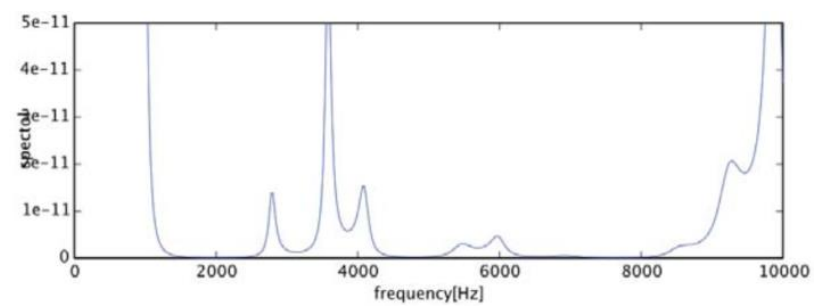

(a)Before

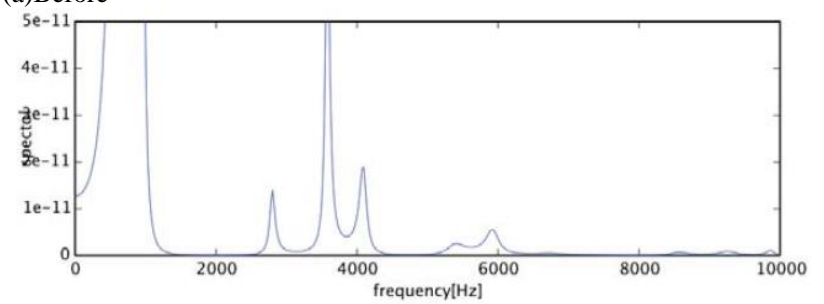

(b)After

Fig. 6. Before and after the low pass filter is applied to the nonlinear equalization multiplier applied voice signals

Also, digital filter featuring wavelet transformation is used for the correction filter. Haar base function of wavelet transformation is used for the first attempt. Haar wavelet transformation is illustrated in Fig.7. The original voice signals in time domain can be converted in high (H1) and low (L1) frequency components as shown in Fig.7. Then L1 component can also be converted in high (H2) and low (L2) components and so on. These components are called as wavelet coefficients (frequency components). Using the wavelet coefficients, Hn and Ln, Ln-1 can be reconstructed perfectly because Haar wavelet function is bi-orthogonal function.

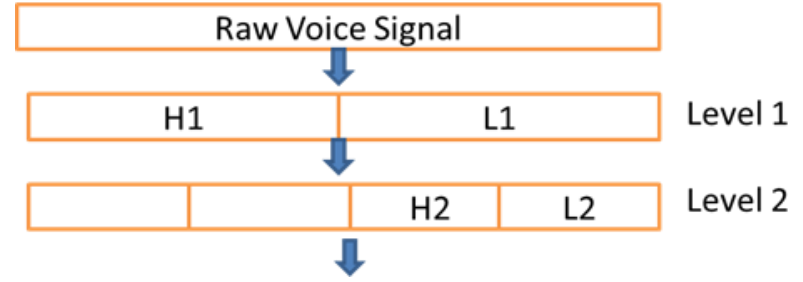

Fig. 7. Haar wavelet trasformation

Then raw input voice signal is converted through Haar wavelet trasformation with level 5 which corresponds to the third formant frequency. After that nonlinear multiplication is applied to the converted wavelet coefficients as shown in Fig.8. Also, the nonlinear multiplication is aplied to the converted wavelet coefficients with the previously designed cut off frequency. In particular, high frequency components sounds so noisy that the frequency components higher than cut off frequency is better to supressed.

\section{\begin{tabular}{ll|l|lll|lllllllllllll}
\hline L & H & L & H & L & H & L & H & L & H & L & H & L & H & L & H
\end{tabular}}

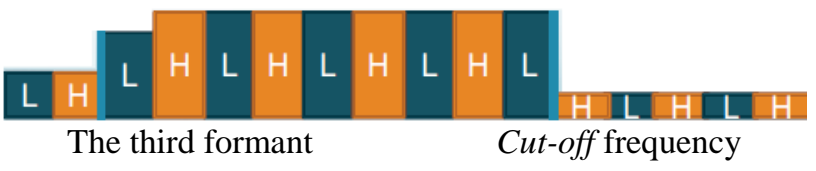

Fig. 8. Concept for the nonlinear equalizer multiplier

It is possible to constract low pass filter based on Haar wavelet transformation. Through reconstraction with the extracted low frequency component only derived from the decomposed voice signals, low pass filter can be realized as shown in Fig.9.

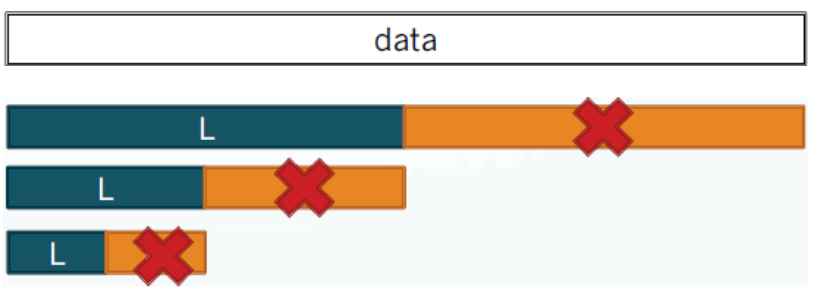

Fig. 9. Low pass filter based on Haar wavelet transformation

Therefore, arbitrary frequency components can be extracted from the level a of the wavelet coeffients. Haar wavelet transformation can be considered as filter bank which allows extraction of arbitrary frequency components. Also, it is possible to reconstruct arbitrary frequency component enhancing voice signals by adding wavelet coefficients. This is the method for frequency component equalization.

Through experiments, the following EEG sensor of ZA-9 + SleepSign-Lite manufactured by Kissei ComTec is used for evaluation of hearing quality. This EEG sensor allows measurements of EEG and electro - oculogram; EOG. Also, voice volume level meter of LM-8102 manufactured by Mother Tool Co. Ltd. is used for the experiments. Outlooks of the EEG and EOG sensor as well as voice volume level meter are shown in Fig. 10 while the major specifications of the EEG and EOG sensors are shown in Table 1. 

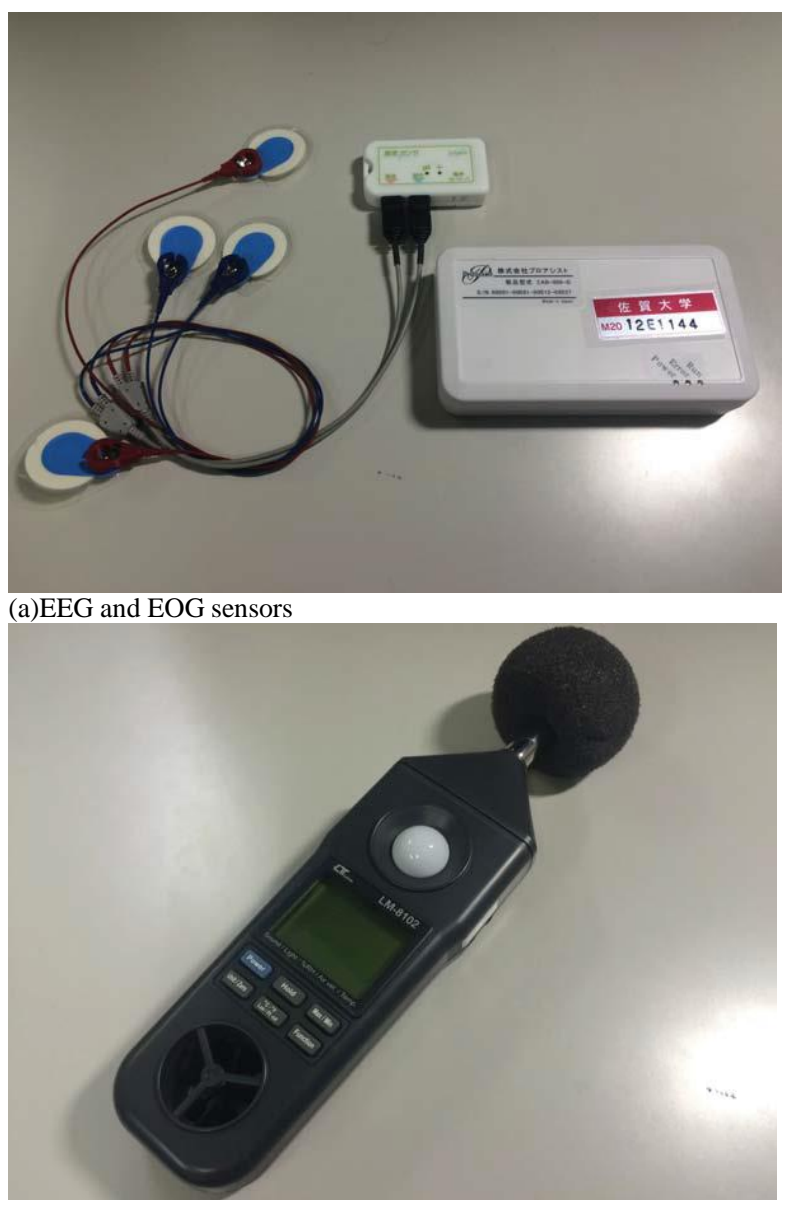

(b) Voice volume level meter

Fig. 10. Outlook of the EEG and EOG sensor as well as voice volume level meter used for the experiments

TABLE I. MAJOR SPECIFICATION OF EEG AND EOG SENSOR OF ZA-9 MANUFACTURED BY KISSEI COMTEC

\begin{tabular}{|l|l|}
\hline Band Width & $0.5 \sim 40 \mathrm{~Hz}$ \\
\hline Sampling Frequency & $128 \mathrm{~Hz}$ \\
\hline AD Converter & 12 bit \\
\hline
\end{tabular}

Meanwhile, the major specification of voice volume level meter is shown in Table 2.

TABLE II. MAJOR SPECIFICATION OF VoICE Volume LEVEL METER OF LM-8102 MANUFACTURED BY MOTHER TOOL CO. LTD

Auto range : $30 \sim 130 \mathrm{~dB}$

Manual range : $\mathrm{L}=30 \sim 80 \mathrm{~dB} / \mathrm{M}=50 \sim 100 \mathrm{~dB} / \mathrm{H}=80 \sim 130 \mathrm{~dB}$

Resolution : $0.1 \mathrm{~dB} \quad$ Frequency range : $31.5 \sim 130 \mathrm{~dB}$

\section{EXPERIMENTS}

Example of input voice signals is shown in Fig.9. From these input voice signals and synthetic voicesignals, formant detection is performed together with creation of characteristics of nonlinear equalizer multiplier for correction of hearing capbility compensation.

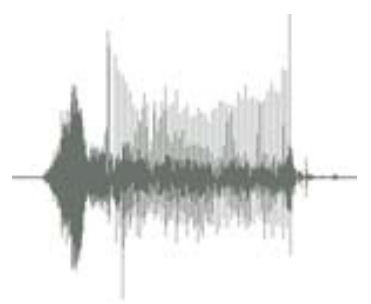

Fig. 11. Example of the input voice signal

The followings are examples of frequencies of the actual and the synthetic formants for "a", " $\mathrm{i}$ " and "u", respectuvely. "a"

F1 :718.75 F2 : 1093.75 F3 :2437.5

$(\mathrm{F} 1=730 ; \mathrm{F} 2=1090 ; \mathrm{F} 3=2440)$ "i")

F1 :289.062 F2 :2296.875 F3:3000

$(\mathrm{F} 1=270 ; \mathrm{F} 2=2290 ; \mathrm{F} 3=3010)$

"u"

F1 :304.688 F2 :882.812 F3 :2226.562

$(\mathrm{F} 1=300 ; \mathrm{F} 2=870 ; \mathrm{F} 3=2240)$

The differences between actual and synthetic formants are very small. Therefore, formants are detected almost perfectly.

In order to determine cut off frequency for noise suppression, the following 67 voice sounds are used.

“a”, “i”, “u”, “e”, “o"

"ka", "ki", "ku", "ke", "ko"

"sa", "si", "su", "se", "so"

"ta", "ti", "tu", "te", "to"

"na", "ni", "nu", "ne", "no"

"ha", "hi", "hu", "he", "ho"

"ma", "mi", "mu", "me", "mo"

"уа", "уи", "уо"

"ra", "ri”, "ru", "re", "ro"

"wa"

"ga", "gi", "gu”, "ge", "go"

"za", "zi", "zu", "ze", "zo"

"da", "di", "du", "de", "d"

"ba", "bi", "bu", "be", "bo"

"pa", "pi", "pu", "pe”, "po"

Percent Correct Recognition of these voice sounds are evaluated with the different cut off frequencies. Nonlinear equalizing multiplier is created depending on the characteristics of hearing capabilities evaluated with EEG and EOG sensors. The results of PCR evaluation is shown in Table 3.

TABLE III. PERCENT CORRECT RECOGNITION: PCR WITH THE DIFFERENT CUT OFF FREQUENCIES

\begin{tabular}{|l|r|r|r|r|r|}
\hline Frequency(Hz) & 689 & 1378 & 2067 & 2756 & 3445 \\
\hline PCR $(\%)$ & 45 & 79 & 90 & 98 & 100 \\
\hline
\end{tabular}

If the cut off frequency is set at $689 \mathrm{~Hz}$, then $55 \%$ of input voice sounds are not recognized. In accordance with the cut 
off frequency, PCR is increased monotonically. PCR reaches $100 \%$ at the cut off frequency of $3445 \mathrm{~Hz}$. Therefore, the first to the third formant frequency have to be maintained their frequency components. Also it may say that 0 to $3443 \mathrm{~Hz}$ of frequency components is mandatory for voice recognitions. Thus it is concluded that cut off frequency has to be set more than $3445 \mathrm{~Hz}$ at least.

According to the investigation of the frequency component analysis and formant detections, most of voice sounds have the formant frequencies for the first to third frequencies within the range of $3445 \mathrm{~Hz}$. Therefore, nonlinear equalizing multiplier is better to enhance the frequency components for the first to third formants in particular. The experimental results with the aforementioned voice input experiments shows that 0 to more than $8000 \mathrm{~Hz}$ of frequency components are required for a good PCR. Also $8162 \mathrm{~Hz}$ cut off frequency would be better for both noise suppressions and keeping a good PCR.

\section{CONCLUSION}

Mobile device based personalized equalizer for improving hearing capability of human voices in particular for elderly persons is proposed. Through experiments, it is found that the proposed equalizer does work well for improving hearing capability by 2 to $55 \%$ of the voice recognition success ratio.

According to the investigation of the frequency component analysis and formant detections, most of voice sounds have the formant frequencies for the first to third frequencies within the range of $3445 \mathrm{~Hz}$. Therefore, the nonlinear equalizing multiplier is better to enhance the frequency components for the first to third formants, in particular. The experimental results with the voice above input experiments show that 0 to more than $8000 \mathrm{~Hz}$ of frequency components are required for a good PCR. Also, $8162 \mathrm{~Hz}$ cut off frequency would be better for both noise suppressions and keeping a good PCR.

\section{ACKNOWLEDGMENT}

The authors would like to thank all the voluntiers of saga University students who participated to the experiments.

\section{REFERENCES}

[1] Bentler Ruth A., Duve , Monica R. (2000). "Comparison of Hearing Aids Over the 20th Century". Ear \& Hearing 21 (6): 625-639. doi:10.1097/00003446-200012000-00009.

[2] Bentler, R. A.; Kramer, S. E. (2000). "Guidelines for choosing a selfreport outcome measure". Ear and hearing 21 (4 Suppl): 37S-49S. doi:10.1097/00003446-200008001-00006. PMID 10981593. edit
[3] Jack Katz; Larry Medwetsky; Robert Burkard; Linda Hood (2009). "Chapter 38, Hearing Aid Fitting for Adults: Selection, Fitting, Verification, and Validation". Handbook of Clinical Audiology (6th ed.). Baltimore MD: Lippincott Williams \& Wilkins. p. 858. ISBN 978-07817-8106-0.

[4] K. Sickel, Shortest Path Search with Constraints on Surface Models of In-ear Hearing Aids 52. IWK, Internationales Wissenschaftliches Kolloquium (Computer science meets automation Ilmenau 10. 13.09.2007) Vol. 2 Ilmenau: TU Ilmenau Universitätsbibliothek 2007, pp. $221-226$

[5] K. Sickel et al., Semi-Automatic Manufacturing of Customized Hearing Aids Using a Feature Driven Rule-based Framework Proceedings of the Vision, Modeling, and Visualization Workshop 2009 (Braunschweig, Germany November 16-18, 2009), pp. 305-312

[6] Dave Fabry, Hans Mülder, Evert Dijkstra (November 2007). "Acceptance of the wireless microphone as a hearing aid accessory for adults". The Hearing Journal 60 (11): 32-36. doi:10.1097/01.hj.0000299170.11367.24.

[7] Hawkins D (1984). "Comparisons of speech recognition in noise by mildly-to-moderately hearing-impaired children using hearing aids and FM systems". Journal of Speech and Hearing Disorders 49 (4): 409. doi:10.1044/jshd.4904.409.

[8] Ricketts T., Henry P. (2002). "Evaluation of an adaptive, directionalmicrophone hearing aid". International Journal of Audiology 41 (2): 100-112. doi:10.3109/14992020209090400.

[9] Lewis M Samantha, Crandell Carl C, Valente Michael, Horn Jane Enrietto (2004). "Speech perception in noise: directional microphones versus frequency modulation (FM) systems". Journal of the American Academy of Audiology 15 (6): 426-439. doi:10.3766/jaaa.15.6.4.

[10] Exemption from Preemption of State and Local Hearing Aid Requirements; Applications for Exemption, Docket No. 77N-0333, 45 Fed. Reg. 67326; Medical Devices: Applications for Exemption from Federal Preemption of State and Local hearing Aid Requirements, Docket No. 78P-0222, 45 Fed. 67325 (Oct. 10, 1980).

\section{AUTHORS PROFILE}

Kohei Aarai He received BS, MS and $\mathrm{PhD}$ degrees in 1972, 1974 and 1982, respectively. He was with The Institute for Industrial Science and Technology of the University of Tokyo from April 1974 to December 1978 and also was with National Space Development Agency of Japan from January, 1979 to March, 1990. During from 1985 to 1987, he was with Canada Centre for Remote Sensing as a Post Doctoral Fellow of National Science and Engineering Research Council of Canada. He moved to Saga University as a Professor in Department of Information Science on April 1990 He was a councilor for the Aeronautics and Space related to the Technology Committee of the Ministry of Science and Technology during from 1998 to 2000. He was a councilor of Saga University for 2002 and 2003. He also was an executive councilor for the Remote Sensing Society of Japan for 2003 to 2005. He is an Adjunct Professor of University of Arizona, USA since 1998. $\mathrm{He}$ also is Vice Chairman of the Commission-A of ICSU/COSPAR since 2008. He wrote 33 books and published 500 journal papers. 